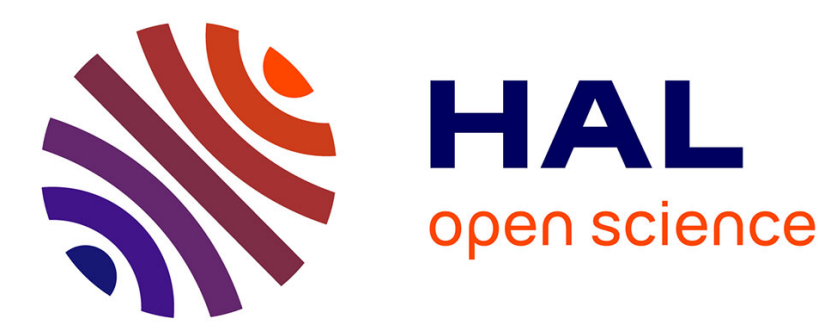

\title{
Pressure broadening of an anticrossing signal
}

\author{
J. Derouard, R. Jost, M. Lombardi
}

\section{To cite this version:}

J. Derouard, R. Jost, M. Lombardi. Pressure broadening of an anticrossing signal. Journal de Physique Lettres, 1976, 37 (6), pp.135-138. 10.1051/jphyslet:01976003706013500 . jpa-00231257

\section{HAL Id: jpa-00231257 https://hal.science/jpa-00231257}

Submitted on 1 Jan 1976

HAL is a multi-disciplinary open access archive for the deposit and dissemination of scientific research documents, whether they are published or not. The documents may come from teaching and research institutions in France or abroad, or from public or private research centers.
L'archive ouverte pluridisciplinaire HAL, est destinée au dépôt et à la diffusion de documents scientifiques de niveau recherche, publiés ou non, émanant des établissements d'enseignement et de recherche français ou étrangers, des laboratoires publics ou privés. 


\title{
PRESSURE BROADENING OF AN ANTICROSSING SIGNAL
}

\author{
J. DEROUARD, R. JOST and M. LOMBARDI
}

Laboratoire de Spectrométrie Physique (*), Université Scientifique et Médicale de Grenoble, B.P. 53, 38041 Grenoble Cedex, France

(Reçu le 8 mars 1976, accepté le 13 avril 1976)

\begin{abstract}
Résumé. - Nous montrons que l'élargissement sous l'effet de la pression de la largeur $2 \sqrt{4 V^{2}+(\hbar \Gamma)^{2}}$ d'un signal d'anticroisement (où $V$ est le couplage entre les deux niveaux qui s'anticroisent et $\Gamma$ l'inverse de leur durée de vie) n'est pas dû seulement à l'accroissement de $\Gamma$ sous l'effet des collisions. Nous montrons que la largeur du signal est donnée par la formule $2 \sqrt{4 V^{2} a^{a b} \gamma / \gamma+\left(\hbar^{a b} \gamma\right)^{2}}$, où ${ }^{a b} \gamma / \gamma$ est le rapport des temps de relaxation effectifs de la cohérence et des populations des deux niveaux.
\end{abstract}

Abstract. - We show that the pressure broadening of the width $2 \sqrt{4 V^{2}+(\hbar \Gamma)^{2}}$ of an anticrossing signal (where $V$ is the coupling between the anticrossing levels and $\Gamma$ the reciprocal of their lifetime) is caused by more than just the increase of $\Gamma$ by collisions. We find that the linewidth is given by $2 \sqrt{4 V^{2} \mathrm{ab}^{\mathrm{b}} / \gamma+\left(\hbar^{\mathrm{ab}} \gamma\right)^{2}}$, where ${ }^{\mathrm{ab}} \gamma / \gamma$ is the ratio of the effective relaxation times of the coherences to the populations of the anticrossing levels.

1. Introduction. - The anticrossing phenomenon occurs whenever two levels $|a\rangle$ and $|\mathbf{b}\rangle$, with unperturbed energies $E_{\mathrm{a}}$ and $E_{\mathrm{b}}$, of an atom or a molecule are brought into near coincidence by applying a magnetic field, provided that they are coupled by some interaction $V=\langle\mathrm{a}|\mathrm{V}| \mathrm{b}\rangle$. In the vicinity of the crossing point, the eigenvalues $E_{+}$ and $E_{-}$of the total Hamiltonian repel one other and the associated eigenfunctions $|+\rangle$ and $|-\rangle$ are mixtures $|\mathrm{a}\rangle$ and $|\mathrm{b}\rangle$. This produces an equalization of the populations of $|a\rangle$ and $|b\rangle$ at the avoided crossing point if some steady state excitation mechanism produces a difference between the populations of $|a\rangle$ and $|b\rangle$. This resonant-like variation of population may be observed by monitoring separately the intensity (and/or polarization) of the light emitted by $|\mathrm{a}\rangle$ and $|\mathrm{b}\rangle$. This phenomenon was observed for the first time on the sublevels of the $2{ }^{2} \mathrm{P}$ manifold of $\mathrm{Li}$ [1]. The coupling was due to the hyperfine interaction. Since then, several other experiments have been carried out in which $v$ was due to an electric field connecting two opposite parity levels [2], and more recently to a fine or hyperfine interaction connecting singlet and triplet levels of helium-like systems [3]. This last kind of experiment has led to the relaxation studies which follow.

We would like to mention from the onset that experiments of magnetic or electric resonance can be

(*) Laboratoire associé au Centre National de la Recherche Scientifique. considered as anticrossing experiments in the space of atom + quantized radiofrequency field [4] so that effects analogous to the one we study here do exist in resonance experiments. One aspect of this similarity is studied in the third paragraph.

The usual calculation of the anticrossing signal for steady state excitation, based on the solution of the following rate equation for the density matrix

$$
i \frac{\mathrm{d} \rho}{\mathrm{d} t}=0=\frac{1}{\hbar}\left(\mathcal{H}_{0}+\mathcal{V}, \rho\right)+\overbrace{\text { pumping }}^{+i N+i \frac{\mathrm{d} \rho}{\mathrm{d} t} \overbrace{\text { radiative decay }}}
$$

with

$$
\begin{aligned}
\mathscr{H}_{0}|\mathrm{a}\rangle & =E_{\mathrm{a}}|\mathrm{a}\rangle, \mathscr{H}_{0}|\mathrm{~b}\rangle=E_{\mathrm{b}}|\mathrm{b}\rangle, \\
\langle\mathrm{a}|\mathcal{V}| \mathrm{a}\rangle & =\langle\mathrm{b}|\mathcal{V}| \mathrm{b}\rangle=0,\langle\mathrm{a}|\mathfrak{V}| \mathrm{b}\rangle=V,
\end{aligned}
$$

is straightforward. In the simplest case in which two levels of same radiative lifetime $1 / \Gamma$ are excited incoherently with rates $n_{\mathrm{a}}$ and $n_{\mathrm{b}}$, one finds that the steady state population of $|\mathrm{a}\rangle$ is

$$
\rho_{\mathrm{a}}=\frac{n_{\mathrm{a}}}{\Gamma}-\frac{2|V|^{2}}{\Gamma} \frac{n_{\mathrm{a}}-n_{\mathrm{b}}}{\left(E_{\mathrm{a}}-E_{\mathrm{b}}\right)^{2}+4|V|^{2}+(\hbar \Gamma)^{2}}
$$

(and the symmetric formula for $\rho_{\mathrm{b}}$ ). This produces, as a function of $\left(E_{\mathrm{a}}-E_{\mathrm{b}}\right)$, a Lorentzian signal of width at half maximum

$$
\Delta E=2 \sqrt{4|V|^{2}+(\hbar \Gamma)^{2}} .
$$


We have performed experiments on singlet triplet anticrossings of ${ }^{4} \mathrm{He}[3]$. In this case $V$, the spin orbit coupling, is roughly 100 times larger than the radiative decay term $\hbar \Gamma$, so that the influence of $\hbar \Gamma$ in (3) on the linewidth is only $10^{-4}$. When one increases the pressure to a few times $10^{-1}$ torr, $\Gamma$ is shortened by collisions by a factor of two or three [5] which, according to formula (3), implies a negligible broadening of the anticrossing curve. In fact, we have found experimentally that the width was roughly doubled. The purpose of this letter is to give the explanation of this phenomenon.

2. Theory. - At high pressure, when the interatomic collisions do play a role, eq. (1), restricted to the two anticrossing levels $|\mathrm{a}\rangle$ and $|\mathrm{b}\rangle$, is no longer sufficient. $|\mathrm{a}\rangle$ and $|\mathrm{b}\rangle$ are two particular Zeeman sublevels, amid two Zeeman sets $\left|\mathrm{a}^{\prime}\right\rangle,\left|\mathrm{a}^{\prime \prime}\right\rangle \ldots$ and $\left|b^{\prime}\right\rangle,\left|b^{\prime \prime}\right\rangle \ldots$ of two levels $a$ and $b$. The collisions produce transfers between Zeeman sublevels of a same level, as well as quenching transfers to other levels. One takes this into account by replacing eq. (1) by :

$$
\begin{aligned}
i \frac{\mathrm{d} \rho}{\mathrm{d} t} & =\frac{1}{\hbar}\left(\mathcal{H}_{0}+\mathcal{V}, \rho\right)+i N+\left.i \frac{\mathrm{d} \rho}{\mathrm{d} t}\right|_{\mathrm{rad}}+\left.i \frac{\mathrm{d} \rho}{\mathrm{d} t}\right|_{\text {coll }} \\
& =\frac{1}{\hbar}\left(\mathcal{H}_{0}+\mathcal{V}, \rho\right)+i N+\left.i \frac{\mathrm{d} \rho}{\mathrm{d} t}\right|_{\mathrm{relax}} .
\end{aligned}
$$

We shall now show with a simplified example how these transfers can produce a great increase of the anticrossing width. Consider two levels $a$ and $b$ of angular momentum $J=\frac{1}{2}$, where $|\mathrm{a}+\rangle,|\mathrm{a}-\rangle$ and $|\mathrm{b}+\rangle,|\mathrm{b}-\rangle$ are their Zeeman sublevels. The sublevels $|\mathrm{a}-\rangle$ and $|\mathrm{b}+\rangle$ are coupled by $V$ and anticross. The other two do not anticross but they are coupled to the anticrossing ones by collision. This is a limiting case of our ${ }^{4} \mathrm{He} n \mathrm{D}$ levels studies [3] in which the five Zeeman sublevels of the D levels are replaced by two.

We make the following assumptions on the collision process, whose validity in our experimental case will be discussed in a forthcoming detailed paper.

- The collisions do not couple $a$ and $b$. This corresponds to the Wigner spin rule in the case of singlet triplet anticrossings. In this case ${ }^{\text {aa }} \rho$, the projection of the density matrix $\rho$ into the subspace $\mathrm{a},{ }^{\mathrm{bb}} \rho$, its projection into $\mathrm{b}$, and ${ }^{\mathrm{ab}} \rho$, which represents the coherence between $\mathrm{a}$ and $\mathrm{b}$ are not coupled by collisions.

- The relaxation process is isotropic, i.e. the anisotropy due to the magnetic field has no influence on the collision process. This amounts to saying that the evolution of the atom under the influence of the Zeeman effect is negligible during the collision time.

One can show [6] that this isotropy hypothesis implies that the relaxation of each ${ }^{\mathbf{x} x^{\prime}} \rho$, with $\mathbf{x}, \mathbf{x}^{\prime}=\mathrm{a}, \mathrm{b}$, depends only on two coefficients ${ }^{x x^{\prime}} \gamma^{(0)}$ and ${ }^{x x^{\prime}} \gamma^{(1)}$. More precisely, introducing the linear combinations of the density matrix components ${ }^{\mathbf{x}} \boldsymbol{\rho}_{ \pm \pm}$which are proportional, within a subspace of spin $\frac{1}{2}$ to the mean values of magnetic moment operators (or Pauli matrices), one has

$$
\begin{aligned}
& \frac{\mathrm{d}\left\langle M_{x}\right\rangle}{\mathrm{d} t} \propto \frac{\mathrm{d}}{\mathrm{d} t}\left({ }^{\mathbf{x x}^{\prime}} \rho_{+-}+{ }^{\mathrm{xx}^{\prime}} \rho_{-+}\right)_{\mathrm{relax}}=-{ }^{\mathbf{x x}^{\prime}} \gamma^{(1)}\left(\mathbf{x x}^{\prime} \rho_{+-}+{ }^{\mathbf{x x}^{\prime}} \rho_{-+}\right) \\
& \frac{\mathrm{d}\left\langle M_{y}\right\rangle}{\mathrm{d} t} \propto \frac{\mathrm{d}}{\mathrm{d} t}\left({ }^{\mathbf{x x}} \rho_{+-}-{ }^{\mathbf{x x}^{\prime}} \rho_{-+}\right)_{\mathrm{relax}}=-{ }^{\mathbf{x x}^{\prime}} \gamma^{(1)}\left({ }^{\mathbf{x x}^{\prime}} \rho_{+-}-{ }^{\mathbf{x x}^{\prime}} \rho_{-+}\right) \\
& \frac{\mathrm{d}\left\langle M_{z}\right\rangle}{\mathrm{d} t} \propto \frac{\mathrm{d}}{\mathrm{d} t}\left({ }^{\mathbf{x x}^{\prime}} \rho_{++}-{ }^{\mathbf{x x}^{\prime}} \rho_{--}\right)_{\text {relax }}=-{ }^{\mathbf{x x}^{\prime}} \gamma^{(1)}\left(\mathbf{x x}^{\prime} \rho_{++}-{ }^{\mathbf{x x}^{\prime}} \rho_{--}\right) \\
& \frac{\mathrm{d}\left\langle\sqrt{\mathbf{M}^{2}}\right\rangle}{\mathrm{d} t} \propto \frac{\mathrm{d}}{\mathrm{d} t}\left({ }^{\mathbf{x}{ }^{\prime}} \rho_{++}+{ }^{\mathbf{x x}} \rho_{--}\right)_{\text {relax }}=-{ }^{\mathbf{x x}} \gamma^{(0)}\left({ }^{\mathbf{x x}} \rho_{++}+{ }^{\mathbf{x x}} \rho_{--}\right) \text {. }
\end{aligned}
$$

In comparison to the relaxation terms usually introduced in the Bloch equations, this shows two differences. First, the transverse and longitudinal relaxation times $T_{1}$ and $T_{2}$ are both equal to $1 / \mathbf{x x}^{\prime} \gamma^{(1)}$, which is a consequence of the isotropy hypothesis.

Second, the modulus of $\mathbf{M}$ is not constant. ${ }^{\mathbf{x x}^{\prime}} \gamma^{(0)}$ takes into account the de-excitation processes, both radiative and collisional (quenching collision transfers to levels others than a and $\mathrm{b}$, which, in the case of the $n \mathrm{D}$ levels of $\mathrm{He}$, are rather important due to the proximity of the $n \mathrm{P}$ and $n \mathrm{~F}$ levels). We shall further simplify the problem by supposing ${ }^{a a} \gamma^{(0)}={ }^{b b_{2}} \gamma^{(0)}=\gamma^{(0)}$ and ${ }^{a a} \gamma^{(1)}={ }^{b b} \gamma^{(1)}=\gamma^{(1)}$, since a full calculation shows that this simplification changes nothing essential. The useful equations are then :

$$
\begin{aligned}
& \frac{\mathrm{d}}{\mathrm{d} t}\left({ }^{\mathbf{x x}} \rho_{++}\right)_{\mathrm{relax}}=-\frac{\gamma^{(0)}+\gamma^{(1)}}{2}{ }^{\mathrm{xx}} \rho_{++}+\frac{\gamma^{(1)}-\gamma^{(0)}}{2}{ }^{\mathbf{x x}} \rho_{--} \\
& \frac{\mathrm{d}}{\mathrm{d} t}\left({ }^{\mathbf{x x}} \rho_{--}\right)_{\text {relax }}=-\frac{\gamma^{(0)}+\gamma^{(1)}}{2}{ }^{\mathbf{x x}} \rho_{--}+\frac{\gamma^{(1)}-\gamma^{(0)}}{2}{ }^{\mathbf{x}} \rho_{++} \\
& \frac{\mathrm{d}}{\mathrm{d} t}\left({ }^{\mathrm{ab}} \rho_{-+}\right)_{\text {relax }}=-{ }^{\mathrm{ab}} \gamma^{(1)}{ }^{\mathrm{ab}} \rho_{-+} .
\end{aligned}
$$


$2 /\left(\gamma^{(0)}+\gamma^{(1)}\right)$ is then the average time during which the atom remains within one Zeeman sublevel, while $1 / \gamma^{(0)}$ is the average time during which it remains within a whole excited level a or b. Usually $\gamma^{(1)} \gg \gamma^{(0)}$ and the first time is considerably shorter than the second. Putting $\left(4^{\prime}\right)$ into $\left(1^{\prime}\right)$ a straightforward calculation leads to

$$
\begin{aligned}
& { }^{\mathrm{aa}} \rho_{--}=\frac{n_{\mathrm{a}}}{\gamma^{(0)}}-\frac{2|V|^{2}}{\gamma^{(0)}} \frac{n_{\mathrm{a}}-n_{\mathrm{b}}}{\left(E_{\mathrm{a}-}-E_{\mathrm{b}+}\right)^{2}+4|V|^{2} \frac{\gamma^{(1)}}{\gamma}+\left(\hbar^{\mathrm{ab}} \gamma^{(1)}\right)^{2}} \frac{\mathrm{ab}_{\gamma^{(1)}}}{\gamma} \\
& { }^{\mathrm{aa}} \rho_{++}=\frac{n_{\mathrm{a}}}{\gamma^{(0)}}-\frac{2|V|^{2}}{\gamma^{(0)}} \frac{n_{\mathrm{a}}-n_{\mathrm{b}}}{\left(E_{\mathrm{a}-}-E_{\mathrm{b}+}\right)^{2}+4|V|^{2} \frac{{ }^{\mathrm{ab}}{ }^{(1)}}{\gamma}+\left(\hbar^{\mathrm{ab}} \gamma^{(1)}\right)^{2}} \frac{{ }^{\mathrm{ab}} \gamma^{(1)}}{\gamma} \frac{\gamma^{(1)}-\gamma^{(0)}}{\gamma^{(1)}+\gamma^{(0)}}
\end{aligned}
$$

and to similar expressions for ${ }^{\mathrm{bb}} \rho_{++}$and ${ }^{\mathrm{bb}} \rho_{--}$, with :

$$
\frac{1}{\gamma}=\frac{1}{2}\left(\frac{1}{\gamma^{(0)}}+\frac{1}{\gamma^{(1)}}\right)
$$

One then finds that, if $\gamma^{(1)} \neq \gamma^{(0)}$ (no pure quenching) the anticrossing signal appears also in $|\mathrm{a}+\rangle$ (and $|\mathrm{b}-\rangle)$. This is a consequence of the Zeeman transfer.

More important, the anticrossing shape remains Lorentzian as a function of $E_{\mathrm{a}-}-E_{\mathrm{b}+}$, but its width is now

$$
\Delta E=2 \sqrt{4|V|^{2} \frac{\mathrm{ab}^{(1)}}{\gamma}+\left(\hbar^{\mathrm{ab}} \gamma^{(1)}\right)^{2}} .
$$

It is the ${ }^{a b} \gamma^{(1)} / \gamma$ term which explains the anomalous increase of the width we have found experimentally.

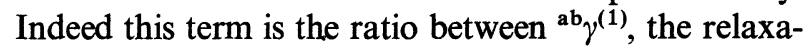
tion time of the coherence between $|\mathrm{a}+\rangle$ and $|\mathrm{b}-\rangle$, and $\gamma$, which is an effective relaxation time of

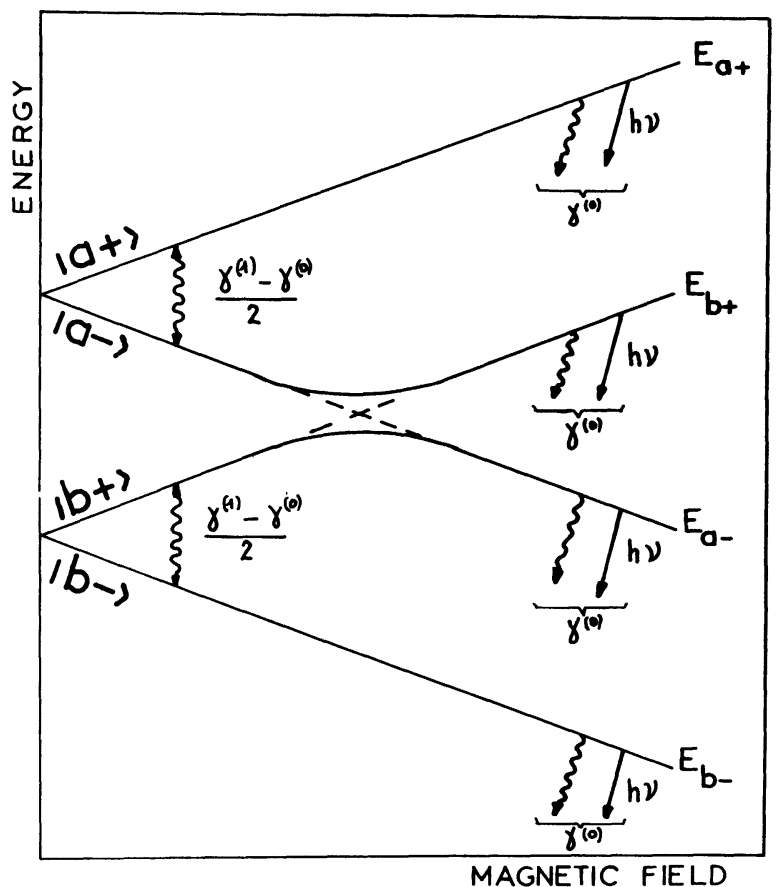

FIG. 1. - Simplified model used to study the effect of relaxation processes on an anticrossing signal. Wiggly lines correspond to collision transfers. the population of these levels. This ratio is equal to 1 at zero pressure for equal lifetimes of $a$ and $b$, but is greater than 1 when there are collisions since a forward-backward transfer between, say, $|\mathrm{a}-\rangle$ and $|\mathrm{a}+\rangle$ brings back the population of $|\mathrm{a}-\rangle$ but not the coherence between $|\mathrm{a}-\rangle$ and $|\mathrm{b}+\rangle$. More precisely, in our experimental case ${ }^{a b} \gamma^{(1)} \sim \gamma^{(1)}$, and we shall take

$$
\begin{aligned}
\mathrm{ab}^{(1)} & =\gamma^{(1)}=(1+P) \Gamma \\
\gamma^{(0)} & =(1+K P) \Gamma,
\end{aligned}
$$

where $P$ is proportional to both the pressiure and the depolarization cross-section, and $K$ is the ratio between the quenching and the depolarization cross-section, usually much smaller than 1 . All of these expressions are valid only one can neglect the back transfer from the quenching levels to $a$ or $b$. Finally one obtains :

$$
\Delta E=2 \sqrt{4|V|^{2} \frac{2+(1+K) P}{2(1+K P)}+(1+P)^{2}(\hbar \Gamma)^{2}} .
$$

The factor which multiplies $4|V|^{2}$ varies from 1 at zero pressure to $(K+1) / 2 \dot{K}$ at high pressure. This last expression is usually $\gg 1$, since $K \ll 1$. Another consequence of the formula (5) is the prediction of a decrease, in the ratio of the polarization to the intensity signal which is independent of the excitation $n_{\mathrm{a}}$ and $n_{\mathrm{b}}$ :

$$
\begin{aligned}
\frac{\left({ }^{\text {aa }} \rho_{++}-\frac{n_{\mathrm{a}}}{\gamma^{(0)}}\right)-\left({ }^{\mathrm{aa}} \rho_{--}-\frac{n_{\mathrm{a}}}{\gamma^{(0)}}\right)}{\left({ }^{\mathrm{aa}} \rho_{++}-\frac{n_{\mathrm{a}}}{\gamma^{(0)}}\right)+\left({ }^{\mathrm{aa}} \rho_{--}-\frac{n_{\mathrm{a}}}{\gamma^{(0)}}\right)} & = \\
& =-\frac{\gamma^{(0)}}{\gamma^{(1)}}=-\left(\frac{1+K P}{1+P}\right) .
\end{aligned}
$$

This depolarization phenomenon has been experimentally verified.

3. Comparison with Bloch's equations. - A better insight on these relaxation phenomena can be obtained by writing down the equations which relate population and coherence of the anticrossing $|\mathrm{a}-\rangle$ and $|\mathrm{b}+\rangle$ 
levels in the case of steady state excitation. We introduce the linear combinations :

$$
\begin{array}{rlrl}
u & ={ }^{\mathrm{ab}} \rho_{-+}+{ }^{\mathrm{ba}} \rho_{+-}, & v & =i\left({ }^{\mathrm{ab}} \rho_{-+}-{ }^{\mathrm{ba}} \rho_{+-}\right), \\
M_{z}={ }^{\mathrm{aa}} \rho_{--}-{ }^{\mathrm{bb}} \rho_{++}, & \sqrt{\mathbf{M}^{2}}={ }^{\mathrm{aa}} \rho_{--}+{ }^{\mathrm{bb}} \rho_{++}
\end{array}
$$

These combinations are analogous to, but different from the expressions (4). In (4) all $\mathrm{x}$ and $\mathrm{x}^{\prime}$ are consistently equal to a and/or $b$, whereas in (7) there are mixed expressions corresponding to the populations of $|\mathrm{a}-\rangle$ and $|\mathrm{b}+\rangle$, and to the coherence between them. Eliminating in $\left(1^{\prime}\right)$ (with $\mathrm{d} \rho / \mathrm{d} t=0$ ) the matrix elements of $\rho$ corresponding to the non anticrossing states $|\mathrm{a}+\rangle$ and $|\mathrm{b}-\rangle$, one obtains (with $V$ real) :

$$
\begin{aligned}
& 0=-v\left(E_{\mathrm{a}-}-E_{\mathrm{b}+}\right)-\mathbf{a b}_{\gamma^{(1)}} u \\
& 0=u\left(E_{\mathrm{a}-}-E_{\mathrm{b}+}\right)-2 V M_{z}-{ }^{\mathbf{a b}_{\gamma^{(1)}}} v \\
& 0=2 V v-\gamma M_{z}+\left(n_{\mathrm{a}}^{\prime}-n_{\mathrm{b}}^{\prime}\right) \\
& 0=\left(n_{\mathrm{a}}^{\prime}+n_{\mathrm{b}}^{\prime}\right)-\gamma \sqrt{\mathbf{M}^{2}}
\end{aligned}
$$

with $n_{\mathrm{x}}^{\prime}=n_{\mathrm{x}} \gamma / \gamma^{(0)}$. The first three equations (8) are identical to the rotating frame steady state Bloch equations for the fictitious spin $\frac{1}{2}$ associated to $|\mathrm{a}-\rangle$ and $|\mathrm{b}+\rangle$, with a detuning

$$
\delta \omega=g \mu_{\mathrm{B}} B_{0}-\omega=E_{\mathrm{a}-}-E_{\mathrm{b}_{+}},
$$

a R.F. field $B_{1}=2 V / g \mu_{\mathrm{B}}$ and a longitudinal time constant $T_{1}=1 / \gamma$ different from the transverse time constant $T_{2}=1 /{ }^{\mathrm{ab}} \gamma^{(1)}$. This does not correspond to a real spatial anisotropy of the relaxation process, as is evident from eq. (4), but to the above-mentioned difference of relaxation times $1 /{ }^{\mathbf{a}} \gamma^{(1)}$ for the coherence between $|\mathrm{a}-\rangle$ and $|\mathrm{b}+\rangle$ (which is the transverse quantity in (7) and (8)) and $1 / \gamma$ for their population difference (which is the longitudinal quantity). The anomalous increase in width of the anticrossing signal is then equivalent to the saturation contribution to the linewidth $2 \sqrt{1 / T_{2}^{2}+\omega_{1}^{2} T_{1} / T_{2}}$ of the Bloch equations.
4. Conclusion. - We finally mention that we have extended this calculation to the pertinent case of the fine structure anticrossings of the $n^{1-3} \mathrm{D}$ levels of ${ }^{4} \mathrm{He}$. This calculation is somewhat cumbersome and will be published later, together with more complete experimental results. An example of the fair agreement between theory and experiment is shown figure 2 . The main point for this letter is that the simple calculation we have outlined here gives all the important results.

We thank the Service National des Champs Intenses of Grenoble (S.N.C.I.), which made possible the experiments reported here.

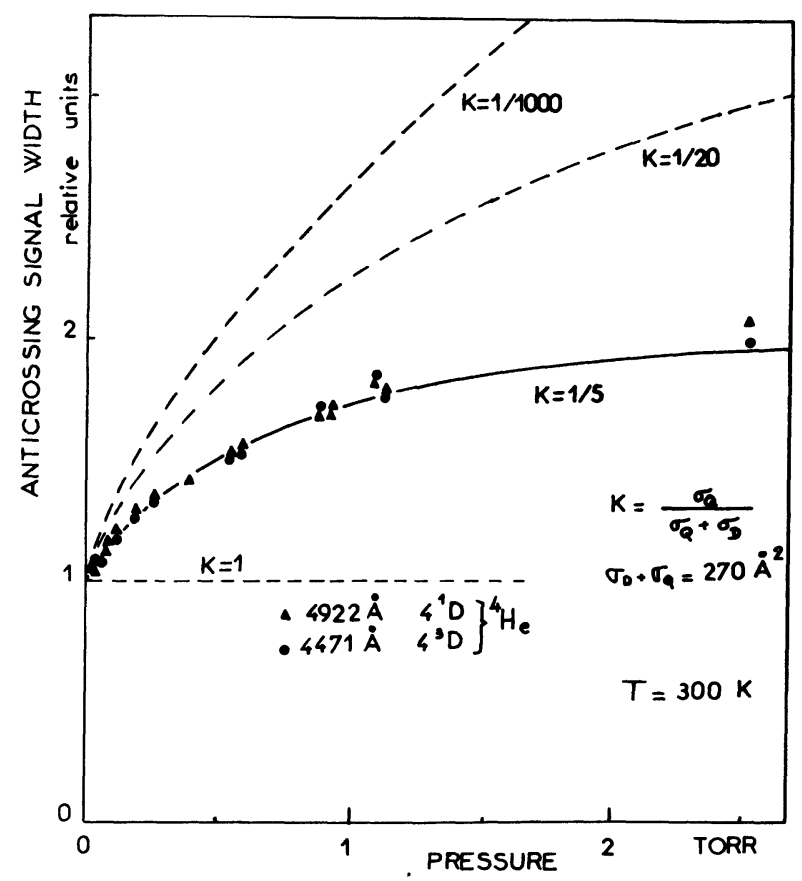

FIG. 2. - Comparison between theory and experiment of the width of the anticrossing signal on the $4{ }^{1} \mathrm{D}$ and $4{ }^{3} \mathrm{D}$ levels of ${ }^{4} \mathrm{He}$. $K=1$ corresponds to what would be expected in the absence of the factor ${ }^{a b} \gamma^{(1)} / \gamma$ in the anticrossing linewidth.

\section{References}

[1] ECK, T. G., Foldy, L. L. and Wieder, H., Phys. Rev. Lett. 10 (1963) 239.

[2] Leventhal, M., Phys. Lett. 20 (1966) 625.

[3] Miller, T. A. and Freund, R. S., J. Chem. Phys. 61 (1974) 2160 .

Jost, R. and Lombardi, M., Phys. Rev. Lett. 33 (1974) 53.

Miller, T. A., Freund, R. S., Tsai, F., Cook, J. J. and Zegarski, B. R., Phys. Rev. A 9 (1974) 2474.

Miller, T. A., Freund, R. S. and Zegarski, B. R., Phys. Rev. A 11 (1975) 753.

Miller, T. A., Freund, R. S., Zegarski, B. R., Jost, R., Lombard, M. and Derouard, J., J. Chem. Phys. 63 (1975) 4042.
Jost, R., Lombardi, M., Derouard, J., Freund, R. S., MiLLer, T. A. and Zegarski, B. R., Chem. Phys. Lett. 37 (1976) 507.

Derouard, J., Jost, R., Lombardi, M., Miller, T. A. and FreUnd, R. S., Phys. Rev. To be published.

[4] Haroche, S., Ann. Phys. 6 (1971) 189 and 327.

[5] Chien, C. W. T., Bardsley, R. E. and Dalby, F. W., Can. J. Phys. 50 (1972) 116.

Pinard, M. and VAN DeR Linde, J., Can. J. Phys. 52 (1974) 1615.

[6] Omont, A., J. Physique 26 (1965) 26.

D'yakonov, M. I. and Perel, V. I., Sov. Phys. JETP 20 (1965) 997.

Omont, A. and Meunier, J., Phys. Rev. 169 (1968) 92. 